Figure 1. Percent of patients treated with antibiotics per culture result

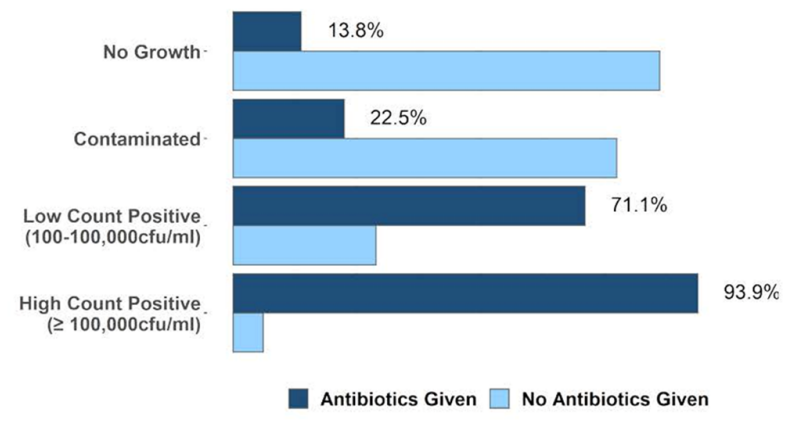

Funding: This study was supported by the National Institute of Allergy and Infectious Diseases of the National Institutes of Health (grant no. UM1AI104681). The content is solely the responsibility of the authors and does not necessarily represent the official views of the National Institutes of Health.

Disclosures: None

Antimicrobial Stewardship \& Healthcare Epidemiology 2021;1(Suppl. S1):s14-s15

doi:10.1017/ash.2021.26

\section{Presentation Type:}

Oral Presentation

Subject Category: Antibiotic Stewardship

Assessing Robustness of Antimicrobial Stewardship in Colorado Hospitals and Barriers to Improvement

Daniel Dodson; Matthew Kronman; Sarah Parker and Christopher Czaja

Background: Adherence to core elements of antimicrobial stewardship programs (ASPs) is increasing nationally but the robustness of programs and inclusion of pediatrics is poorly understood. We describe the details of ASP in Colorado hospitals and identify steps by which academic centers and public health departments can assist community ASPs. Methods: We invited ASP leaders at the 102 acute-care hospitals (ACHs) and critical-access hospitals (CAHs) in Colorado to participate in a web-based survey regarding their ASPs. Questions related to adherence to Centers for Disease Control and Prevention (CDC) core elements, barriers to improvement, desired resources, and extension to pediatrics. Enrollment began in August 2020. Hospital types were compared using the Fisher exact test. Results: As of January 1, 2021, 31 hospitals (30\% of targeted hospitals) completed the web-based survey including $19 \mathrm{ACH}$ and $12 \mathrm{CAH}$. Hospitals were distributed across the state. Median number of beds was 52 (range, 11-680). Of the responding hospitals, $87 \%$ were adherent to all CDC core elements. However, if action was defined as prospective audit and feedback or prior authorization, tracking was defined as measuring

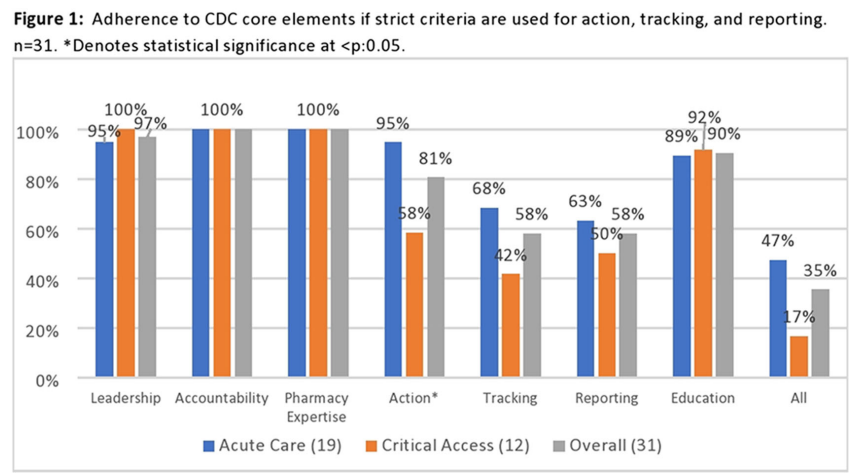

Figure 2: Barriers to providing antibiotic use reports to individual prescribers at hospitals not already providing such reports. $n=26$
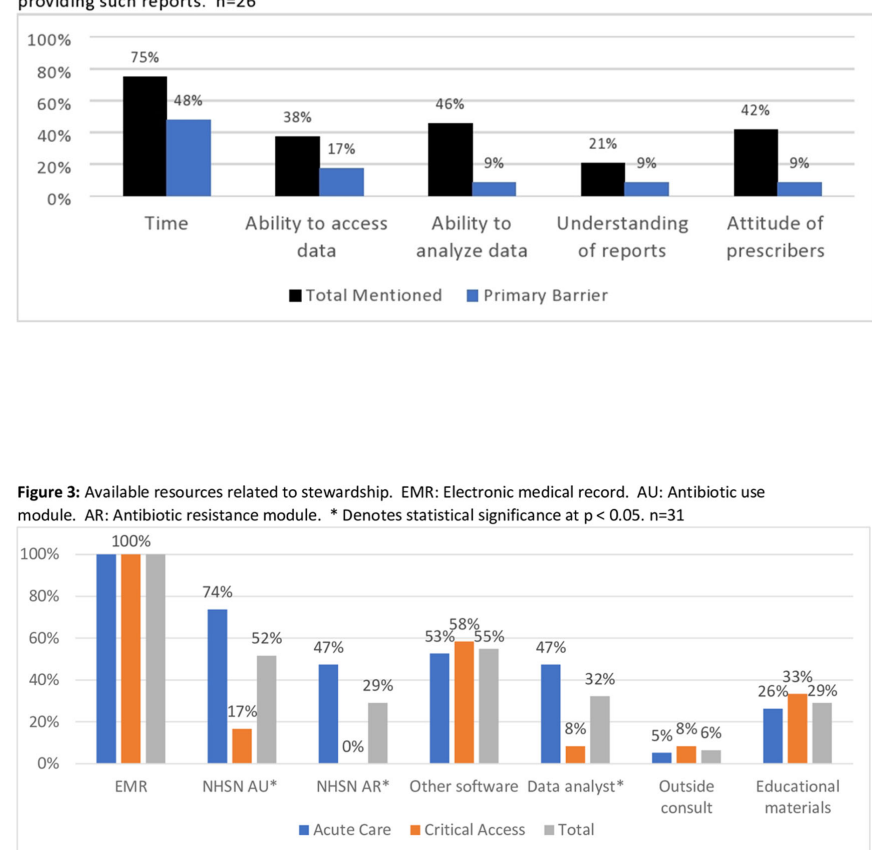

antibiotic use in days of therapy (DOT) or defined daily dose (DDD) quarterly, and reporting was defined as providing unit- or provider-specific antibiotic use reports annually. Overall adherence fell to 35\% including $81 \%$ for action, $58 \%$ for tracking, and $58 \%$ for reporting. CAHs were less likely to adhere to these strict criteria than ACHs (Figure 1). In the 27 hospitals ( $87 \%$ of hospitals) caring for pediatric patients, adherence to a strict action for at least 1 pediatric population was 59\%. Reported barriers to improved ASP were available time and personnel, information technology support, perceived concerns about provider attitudes, and education gaps (Figure 2). CAHs were less likely to use the NHSN antibiotic use or resistance modules or have a data analyst than ACHs (Figure 3). Pediatric pharmacy expertise and guidelines were often not available in hospitals caring for pediatric patients. Desired ASP resources included assistance with data analysis, access to stewardship expertise and education, and treatment guidelines, including for pediatrics. Conclusions: Adherence to CDC core elements of an ASP was excellent but fell dramatically when stricter criteria were used and was worse in pediatric patients. Academic centers and public health departments can assist community hospitals by providing educational resources, assistance in analyzing data including using the NHSN ED: /AR modules, and ASP expertise and clinical care guidelines including those for pediatrics.

Funding: No

Disclosures: None

Antimicrobial Stewardship \& Healthcare Epidemiology 2021;1(Suppl. S1):s15

doi:10.1017/ash.2021.27

\section{Presentation Type:}

Oral Presentation

Subject Category: Antibiotic Stewardship

Interrupted Time-Series Analysis to Evaluate the Impact of a Behavioral Change Outpatient Antibiotic Stewardship Intervention

Brittany Morgan; Larissa May and Haylee Bettencourt

Background: The Centers for Disease Control and Prevention (CDC) estimates that outpatient settings account for $85 \%-90 \%$ of antibiotic prescriptions in the United States, and $\sim 30 \%$ of those prescriptions are unnecessary. One of the most common examples of inappropriate prescribing is for viral upper respiratory infections (URIs). Up to $50 \%$ of prescriptions written for URIs are deemed inappropriate, making it an important focus for Antibiotic Stewardship programs. In this study, we

(C) The Author(s), 2021. Published by Cambridge University Press on behalf of The Society for Healthcare Epidemiology of America. This is an Open Access article, distributed under the terms of the Creative Commons Attribution licence (http://creativecommons.org/licenses/by/4.0/), which permits unrestricted re-use, distribution, and reproduction in any medium, provided the original work is properly cited. 
evaluated the effect of a behaviorally enhanced quality improvement intervention in reducing inappropriate antibiotic prescribing for viral URIs. Methods: A quasi-experimental study assessed the effects of an Antibiotic Stewardship intervention on antibiotic prescribing for viral URIs. The outcome of interest was a change in the number of antibiotics prescribed at each participating clinic over a 1-year preimplementation period and a 2-year postimplementation period. Time trends were analyzed using segmented regression analysis, and a stepped wedge design was used to account for intervention roll-out across clinics. Results: From 2017 to 2020, there were 63,028 patient visits in 21 clinic locations. Antibiotics were prescribed an average of $11.5 \%$ and $5.8 \%$ of visits during the pre- and postimplementation periods, respectively. The most frequently prescribed antibiotic over the study period was azithromycin (n $=3,551)$, followed by amoxicillin $(n=924)$. Overall, the intervention was associated with a $46 \%$ reduction in antibiotic prescriptions or 0.54 times $(P=.001)$ as many inappropriate antibiotics prescribed as before the intervention. There was no significant change in the month-to-month trend in inappropriate prescriptions after the intervention was implemented $(P=.87)$. Conclusions: Our study demonstrates that a behaviorally enhanced quality improvement intervention to reduce inappropriate prescribing for URI in ambulatory care encounters was successful in reducing potentially inappropriate prescriptions for presumed viral respiratory infections.

Funding: No

Disclosures: None

Antimicrobial Stewardship \& Healthcare Epidemiology 2021;1(Suppl. S1):s15-s16

doi:10.1017/ash.2021.28

\section{Presentation Type:}

Oral Presentation

Subject Category: Construction

Bat Intrusions at a Tertiary Care Center, Iowa 2018-2020

Mohammed Alsuhaibani; Takaaki Kobayashi; Lorinda Sheeler; Alexandra Trannel; Stephanie Holley; Oluchi Abosi; Kyle Jenn; Holly Meacham; William Etienne; Angie Dains; Mary Kukla; Bill Millard; Alexandre Marra; Melanie Wellington; Daniel Diekema and Jorge Salinas

Background: Bats are recognized as important vectors in disease transmission. Frequently, bats intrude into homes and buildings, increasing the risk to human health. We describe bat intrusions and exposure incidents in our hospital over a 3-year period. Methods: The University of Iowa Hospitals and Clinics (UIHC) is an 811-bed academic medical center in Iowa City, Iowa. Established in 1928, UIHC currently covers 209,031.84 $\mathrm{m}^{2}$ $\left(\sim 2,250,000 \mathrm{ft}^{2}\right)$ and contains 6 pavilions built between 1928 and 2017 . We retrospectively obtained bat intrusion calls from the infection prevention and control program call database at UIHC during 2018-2020. We have also described the event management for intrusions potentially associated with patient exposures. Results: In total, 67 bat intrusions occurred during 2018-2020. The most frequent locations were hallways or lounges 28 (42\%), nonclinical office spaces 19 (14\%), and stairwells 8 (12\%). Most bat intrusions (65\%) occurred during the summer and fall (June-November). The number of events were 15 in 2018, 28 in 2019, and 24 in 2020. We observed that the number of intrusions increased with the age of each pavilion (Figure 1). Of 67 intrusions, 2 incidents (3\%) were associated with potential exposure to patients. In the first incident, reported in 2019, the bat was captured in a patient care area and released before an investigation of exposures was completed and no rabies testing was available. Also, 10 patients were identified as having had potential exposure to the bat. Among them, 9 patients (90\%) received rabies postexposure prophylaxis. In response to this serious event, we provided facility-wide education on our bat control policy, which includes the capture and safe handling of the bat, assessment of potential exposures, and potential need for rabies testing. We also implemented a bat exclusion project focused on the exterior of the oldest hospital buildings. The second event, 1 patient was identified to have potential exposure to the bat. The bat was captured, tested negative for rabies, no further action was needed. Conclusions: Bat intrusions can be an infection prevention and control challenge in facilities with older buildings.
Bat Intrusions and Year of Hospital Pavilion Construction, Tertiary Care Center, lowa 2018-2020

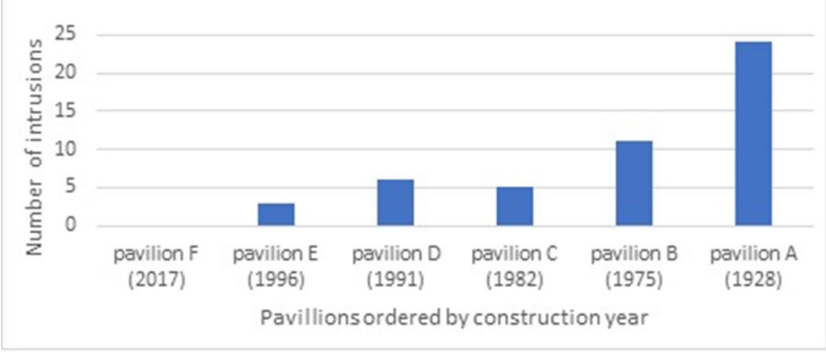

Figure 1.

Hospitals may need animal intrusion surveillance systems, management protocols, and remediation efforts.

Funding: No

Disclosures: None

Antimicrobial Stewardship \& Healthcare Epidemiology 2021;1(Suppl. S1):s16

doi:10.1017/ash.2021.29

Presentation Type:

Oral Presentation

Subject Category: COVID-19

Laboratory-Confirmed SARS-CoV-2 Following One or More Doses of mRNA Vaccine Among Care Team Members at an Academic Health System

Scott Curry; Cassandra Salgado; Krutika Kuppalli; Patricia Nickerson; Morgan Schrader; Danielle Scheurer and Julie Hirschhorn

Background: Medical University of South Carolina Health began vaccinating care team members December 15, 2020, with the Pfizer-BioNTech SARS-CoV-2 mRNA vaccine. We report surveillance data for cases diagnosed following vaccination. Methods: Care team members (CTMs) diagnosed with COVID-19 following SARS-CoV-2 vaccination were selfidentified during online electronic contact-tracing surveys. Demographic data, symptoms, and dates of symptoms were recorded. CTMs testing positive at MUSC were linked to viral burden data from nasopharyngeal swabs tested on Abbott PCR platforms. Results: As of January 31, 2021, 111 CTMs tested positive for SARS-CoV-2 following vaccination: 99 and 12 after 1 and 2 doses, respectively, at medians of 10 days (range, 1-22) and 5 days (range, 1-16), respectively, from vaccination to testing. Of 2 cases that tested positive $>14$ days from dose 2, CTMs had symptom onset at 4 and 12 days from dose 2. Among CTMs reporting symptoms, 104 did so after a median of 7 days (mean 6.3, range -23 to +22) from vaccination to symptom onset, with 8 reporting symptoms before vaccination, 9 on the day of vaccination, and 3 CTMs at 1 day after vaccination, 6 CTMs at 2 days after vaccination, and 11 CTMs at 3 days after vaccination. Overall, $86(78 \%)$ of 111 were female and $95(86 \%)$ of 111 were white. The median age was 44 years (range, 22-71). Clinical job roles were most frequently nurses $(\mathrm{n}=31,28 \%)$, physicians or physician extenders $(\mathrm{n}=19,17 \%)$, and CTMs with no patient contact $(\mathrm{n}=21$, $19 \%)$. Assessment by the contact-tracing team assigned sources as household clusters $(n=22,23 \%)$, local transmission $(n=21,22 \%)$, occupational acquisition from coworkers $(n=11,12 \%)$, travel related $(n=9,10 \%)$, and unknown $(n=32,34 \%)$. Only 32 (32\%) CTMs were compliant with physical distancing. Among 104 CTMs reporting symptoms, cough (75\%), headache $(71 \%)$, rhinorrhea $(63 \%)$, myalgia $(60 \%)$, sore throat $(48 \%)$, anosmia (44\%), and subjective fever (40\%) were the most commonly reported symptoms. Among 54 symptomatic CTMs with available viral-load data, the median and mean cycle numbers $(\mathrm{Cn})$ were 19.98 and 21.91, respectively, for samples tested a median 3 days from symptom onset. Asymptomatic and symptomatic CTMs had a median Cn of 30.1 vs 20.9 , respectively $(\mathrm{p}<0.001)$ and overall $50 \%$ of vaccinated CTMs had $\mathrm{Cn}>20$, with no significant effect seen by vaccine dose (Figure 1). 